\title{
Estudo morfológico e molecular da mama de ratas castradas tratadas com isoflavona ou estrogênios
}

\author{
Morphological and molecular effects of isoflavone and estrogens on the rat mammary gland \\ Ana Claudia Piovesan ${ }^{1}$, José Maria Soares Júnior ${ }^{2}$, Rejane Mosquette ${ }^{3}$, Manuel de Jesus Simões ${ }^{4}$, \\ Ricardo dos Santos Simões ${ }^{5}$, Edmund Chada Baracat ${ }^{6}$
}

\section{RESUMO}

Objetivo: avaliar os efeitos das isoflavonas e dos estrogênios sobre a morfologia, a morfometria e a expressão do fator de crescimento vascular (VEGF) na mama de ratas adultas. Métodos: Quarenta e cinco ratas, após 28 dias de ooforectomia, foram divididas em três grupos: $\mathrm{CON}$ - controle e os grupos que receberam medicação: ISO - isoflavona $(100 \mathrm{mg} / \mathrm{Kg})$ e ECE - estrogênios conjugados eqüinos $(50 \mu \mathrm{g} / \mathrm{Kg})$. Após 60 dias, parte das mamas foram fixadas em formol a $10 \%$ para processamento histológico, e outra parte congelada para a análise da expressão do VEGF pela técnica da reação em cadeia da polimerase com transcrição reversa (RT-PCR). Para análise dos resultados obtidos foram utilizados inicialmente a análise de variância (ANOVA). Quando houve significância, foi complementada com o teste de comparações múltiplas de TukeyKramer. Resultados: Nos grupos CON e ISO as mamas apresentaram glândulas mamárias atróficas, sendo mais desenvolvidas no grupo ECE, onde se notou a presença de inúmeros ductos e de alvéolos mamários. A morfometria mostrou maior número de alvéolos no grupo $\mathrm{ECE}\left(12,3 \pm 7,1^{*}\right.$ por $\left.\mathrm{mm}^{2} ; \mathrm{p}<0,05 \%\right)$ do que nos outros grupos $(\mathrm{CON}=1,4 \pm 2,1$ e ISO =1,6 $\pm 3,8)$, sendo o volume dessas células maior $\left(\mathrm{ECE}=27,4 \pm 9,7 \mu \mathrm{m}^{3}, \mathrm{p}<0,05 \%\right)$ do que nos grupos $\mathrm{CON}\left(14,9 \pm 4,9 \mu \mathrm{m}^{3}\right)$ e ISO $(11,4 \pm 6,9$ $\left.\mu \mathrm{m}^{3}\right)$. O mesmo ocorreu em relação ao número de vasos sanguíneos $\left[16,4 \pm 1,5\right.$ por $\mathrm{mm}^{2}(\mathrm{CON}), 18,4 \pm 2,1$ (ISO) $<37,1 \pm 4,1^{*}$ (ECE), $\mathrm{p}<0,05 \%$ ]. Na análise do VEGF o grupo ECE apresentou maior expressão do que os grupos CON e ISO. Conclusão: nossos dados não evidenciaram efeito proliferativo no tecido mamário de ratas ooforectomizadas tratadas com isoflavona na dose de $100 \mathrm{mg} / \mathrm{Kg}$ durante 60 dias consecutivos.

PALAVRAS-CHAVE: Mama; Estrógenos; Isoflavona; Morfologia; Ratos

\section{ABSTRACT}

Purpose: to analyze the effects of isoflavones and estrogens on the morphology, morphometry and VEGF expression of the adult female rat mammary gland. Methods: Forty-five adult female rats were oophorectomized; 28 days after surgery they were divided into 3 groups of 15 animals each: CON - control (treated with propylenoglycol); ISO - isoflavones (100 mg/kg) and CEE - conjugated equine estrogens $(50 \mu \mathrm{g} / \mathrm{Kg})$. Drugs or vehicle were given orally once a day for 60 days. After this, the animals were killed and the first pair of inguinal mammary glands was immediately removed; part of the material was processed for routine histological study and the remaining tissue was frozen for further analyses of the expression of VEGF mRNA by means of the RT-PCR technique. Results: We observed that mammary ducts were atrophic in the control (CON) and isoflavonetreated (ISO) groups. In these groups the mammary glands were composed of a large concentration of adipose tissue with some ducts and rare alveolar structures. In the CEE group the ducts were well developed with many buds and alveolar structures. The number of mammary gland alveoli was higher in $\mathrm{CEE}$ than in the other groups $(\mathrm{CON}=1.4 \pm 2.1 ; \mathrm{ISO}=1.6 \pm 3.8 ; \mathrm{CEE}=12.3$ \pm 7.1 alveoli $\left./ \mathrm{mm}^{2} ; \mathrm{p}<0.05 \%\right)$; also, the cell volume was higher $\left(\mathrm{CON}=14.9 \pm 4.9 ; \mathrm{ISO}=11.4 \pm 6.9 ; \mathrm{CEE}=27.4 \pm 9.7 \mu \mathrm{m}^{3}, \mathrm{p}<0.05 \%\right)$. The same was observed with regard to the number of blood vessels $(\mathrm{CON}=16.4 \pm 1.5 ; \mathrm{ISO}=18.4 \pm 2.1 ; \mathrm{CEE}=37.1 \pm 4.1 \mathrm{vessels} /$ $\left.\mathrm{mm}^{2}, \mathrm{p}<0.05\right)$. The expression of VEGF in the CEE group was higher than in the other groups, which did not significantly differ from each other in this respect. Conclusion: Our data did not show any proliferation effect in the mammary tissue of adult oophorectomized rats treated with isoflavones $(100 \mathrm{mg} / \mathrm{kg})$ during 60 days.

KEYWORDS: Breast; Estrogens; Isoflavone; Morphology; Rats

\footnotetext{
Universidade Federal de São Paulo - Escola Paulista de Medicina - UNIFESP - São Paulo (SP).

1 Graduanda; Bolsista do Programa Institucional de Bolsa de Iniciação Científica do (PIBIC) do CNPq

2 Professor Afiliado do Departamento de Ginecologia - UNIFESP - São Paulo (SP).

3 Pós-Graduanda do Departamento de Morfologia - UNIFESP - São Paulo (SP).

4 Professor Associado do Departamento de Morfologia - UNIFESP - São Paulo (SP)

5 Médico Residente do Departamento de Ginecologia - UNIFESP - São Paulo (SP).

6 Professor Titular do Departamento de Ginecologia - UNIFESP - São Paulo (SP).

Correspondência: José Maria Soares Júnior

Rua Borges Lagoa, 512, apto. 152c - 04038-000 - São Paulo - SP - e- mail: baracat.gineco@epm.br

Trabalho financiado pela FAPESP (Processo $n^{\circ} 03 / 13407-0$ )

Recebido em: 24/1/2005 Aceito com modificações em: 4/4/2005

Rev Bras Ginecol Obstet. 2005;27(4): 204-9
} 


\section{Introdução}

A melhora das condições de saúde pública levou ao aumento da expectativa de vida. Assim, número cada vez maior de mulheres alcança o climatério. Nessa fase, há alterações fisiológicas e clínicas decorrentes do hipoestrogenismo, como fogachos, sudorese, nervosismo, irritabilidade, insônia, cefaléia, vertigem, depressão, labilidade emocional, diminuição da capacidade de memorização, parestesias, formigamento, palpitações, mialgias, artralgias, atrofia urogenital e diminuição da libido ${ }^{1,2}$.

Para diminuir esta sintomatologia, tem-se utilizado a terapia hormonal $(\mathrm{TH})$ com esteróides sexuais representados predominantemente pelos estrogênios isoladamente ou em associação com progestagênios. A TH tem alguns beneficios para a mulher climatérica, tais como diminuição dos sintomas, melhora da atrofia urogenital e prevenção do desenvolvimento da osteoporose. Além disso, alguns autores mostram que os estrogênios podem aumentar a atividade neuronal, o número de sinapses e retardar o início da doença de Alzheimer ou até diminuir o risco desta afecção ${ }^{3,4}$.

Contudo, a TH apresenta também aspectos negativos, tais como aumento do risco relativo de neoplasias hormônio-dependentes, como no endométrio e na mama, bem como o aumento do risco de tromboembolismo ${ }^{3,5}$. Além disso, algumas pacientes podem apresentar outros efeitos colaterais como mastalgia, aumento de peso corpóreo, alteração do perfil lipídico com aumento do LDL colesterol, principalmente em relação ao progestagênio ${ }^{6}$.

Devido às complicações da terapia estroprogestativa, alguns autores estão investigando novos fármacos que possam oferecer os beneficios semelhantes ao estrogênio no climatério, entre eles as isoflavonas, que são um grupo de fitoestrogênios cuja estrutura molecular tem semelhança bioquímica com o $17 \beta$-estradiol, podendo se ligar aos receptores estrogênicos. Funcionalmente, podem apresentar atividade estrogênica e antiestrogênica, dependendo dos órgãos alvos envolvidos $^{7-9}$.

Estudos clínicos mostraram que as isoflavonas levam a maior conversão de estradiol em estrogênio hidroxilado, mas reduzem os niveis de $17 \alpha$-hidroxiestrona, que é estimulador do câncer de mama. Estudos indicam que os fitoestrogênios reduzem também a bioatividade dos estrogênios, ocupando seus receptores, produzindo efeito antiestrogênico ${ }^{10,11}$.

Há autores que contestam a ação protetora das isoflavonas. Esses advogam que mulheres com dieta rica em isoflavonas (altas doses) poderiam ter proliferação de lesões pré-malignas e aumento de risco de câncer de mama, sendo que o tempo de exposição seria o determinante para esse efeito ${ }^{12}$.

O objetivo deste estudo foi avaliar os efeitos das isoflavonas sobre a morfologia e morfometria da mama de ratas adultas castradas e analisar a expressão do VEGF no tecido mamário, visto ser a literatura escassa e controversa no assunto.

\section{Métodos}

Foram utilizadas 45 ratas adultas (Rattus norvegicus albinus), virgens, de aproximadamente três meses e peso médio de 180 a 210 gramas, fornecidas pelo Centro de Desenvolvimento de Modelos de Experimentação (CEDEME) da Universidade Federal de São Paulo - Escola Paulista de Medicina. Este projeto foi aprovado pelo comitê de Ética da Universidade Federal de São Paulo (Projeto $\mathrm{n}^{\circ}$ 091/03).

Após período de adaptação de duas semanas, todos os animais foram submetidos a exame colpocitológico por sete dias consecutivos e os que apresentaram ciclos estrais normais foram submetidos a ooforectomia bilateral. Para tanto, as ratas foram anestesiadas previamente por via intraperitoneal com cetamina $(100 \mathrm{mg} / \mathrm{kg})$ e xilazina $(20 \mathrm{mg} / \mathrm{kg})$.

Após 28 dias do ato cirúrgico, os animais foram divididos ao acaso em três grupos iguais: $\mathrm{CON}$ - controle (propilenoglicol); ISO - isoflavona (100 $\mathrm{mg} / \mathrm{kg}$, por dia) ${ }^{13}$ e ECE - estrogênios conjugados eqüinos $\left(50 \mu \mathrm{g} / \mathrm{kg}\right.$, por dia) ${ }^{14}$ (Wyeth-Ayerst Laboratories). A composição da isoflavona foi a seguinte: $52 \%$ de isoformas da genisteína; $40 \%$ de isoformas da daidzeína e $8 \%$ de isoformas de glicetina $\left(\right.$ Novasoy $\left.^{\circledR}\right)$. A administração dos fármacos foi realizada por via oral (gavagem), durante 60 dias consecutivos. Após esse período, todos os animais foram anestesiados e sacrificados. Fragmentos do primeiro par de glândulas mamárias inguinais foram removidos e colocados em formol a 10\% para processamento histológico. Outros fragmentos da mesma região mamária foram imediatamente congelados em nitrogênio líquido para extração de RNA mensageiro.

Para rotina histológica, após 24 horas de fixação, os fragmentos mamários foram desidratados em etanol e processados para inclusão em parafina. Os blocos foram submetidos a microtomia, sendo realizados cortes de $3 \mu \mathrm{m}$ de espessura. Quatro lâminas de cada animal foram 
então submetidas ao método de coloração pela hematoxilina e eosina (H.E.).

Para o estudo morfométrico, efetuou-se a captura e digitalização de imagens, com o auxilio de um programa de análise gráfica por computador Imagelab (Softium). Esta parte do trabalho foi realizada no Laboratório de Biologia Molecular do Departamento de Ginecologia da Universidade Federal de São Paulo. As imagens digitalizadas foram analisadas por subtração de cores, separadas por colorimetria, quanto ao volume das células do epitélio alveolar. Já a quantificação do número de alvéolos e de vasos sanguíneos foi realizada por contagem manual em vários campos, perfazendo área de $1 \mathrm{~mm}^{2} \mathrm{em}$ cada corte histológico.

Os fragmentos das mamas mergulhados em nitrogênio líquido foram homogeneizados em TRIzol ${ }^{\circledR}$ Reagent (Life Technologies, Grand Island, NY, USA) e seguiu-se a extração de RNA em todos os grupos para a transcrição reversa combinada à reação em cadeia da polimerase (RT-PCR).

Para a síntese do DNA complementar (DNAc) foram utilizados $8,0 \mu \mathrm{g}$ de RNA total, extraído de cada amostra. As amostras foram incubadas com $1,0 \mu \mathrm{g} / \mathrm{ml}$ de oligo dT a $65^{\circ} \mathrm{C}$ por 5 minutos, para se obter a primeira fita de DNAc. O DNAc obtido foi utilizado como molde nas reações de PCR. Para estes experimentos, foram utilizados primers específicos para o gene do fator de crescimento vásculo-endotelial (VEGF) e como controle o da beta actina. A Tabela 1 mostra a seqüência e o tamanho dos produtos de amplificação, obtidos a partir da reação de RT-PCR.

A partir de uma amostra de RNA do pool de cada grupo, realizamos a reação da transcrição reversa do kit Superscript ${ }^{\mathrm{TM}}$ II RNAASE H reverse transcriptase (Life Technologies).

A reação foi realizada por meio da amplificação, por PCR, ao longo de 45 ciclos em termociclador (MJ Research PTC-200 Thermocycles, Watertown, MA, USA) utilizando-se o kit Master Mix (Eppendorf ${ }^{\circledR}$ ). Os produtos de PCR assim obtidos foram submetidos à eletroforese em gel de agarose e brometo de etídio, sendo a intensidade luminosa das bandas calculada por meio do software Kodak Digital Science - EDAS $120\left(\right.$ Kodak $\left.^{\circledR}\right)$.

Para análise dos resultados obtidos foi utilizada inicialmente a análise de variância (ANOVA). Quando houve significância, foi complementada com o teste de comparações múltiplas de Tukey-Kramer, com o propósito de observar se ocorreram diferenças significantes entre os grupos. Foi fixado em 0,05 ou $5 \%(p \leq 0,05)$ o nivel de significância.

Tabela 1 - Primers utilizados nas reações de RT-PCR. Os tamanhos esperados dos produtos de amplificação em pares de base estão apresentados baseados na sequêencia do DNAc do gene.

\begin{tabular}{lcccc}
\hline Iniciador & Anelamento & GeneBank & Produto & Seqüência \\
\hline VEGF & $58^{\circ} \mathrm{C}$ & NM_031836 & $568 \mathrm{pb}$ & S: 5'-tgc acc cac gac aga agg aga - 3' \\
& & & AS: 5'- tca ccg cct tgg ctt gtc aca t -3' \\
Beta-actina & $55^{\circ} \mathrm{C}$ & V01217 & S: 5'-ttc ctg ggt aag ttg tag tc - 3' \\
& & & AS: 5'- agc act gtg ttg gca tag ag -3'- \\
\hline
\end{tabular}

$\mathrm{S}=$ sense, $\mathrm{AS}=$ antisense, $\mathrm{VEGF}=$ vascular endothelial growth factor.

\section{Resultados}

\section{Morfológicos}

Nos grupos CON e ISO as mamas estavam representadas basicamente por ductos mamários imersos em grande concentração de tecido adiposo. Os ductos mamários apresentaram-se constituídos por duas ou mais camadas de células epiteliais rodeadas por fibras colágenas (Figura 1 - CON e ISO). Em alguns locais pôde-se notar a presença de raros alvéolos mamários, os quais são formados por apenas uma camada de células epiteliais. Deve ser mencionado que no grupo ISO identificaram-se alguns ductos e alvéolos com lúmen dilatado. No grupo ECE notou-se que a mama estava constituída por inúmeros ductos e alvéolos mamários. Os ductos apresentaram-se constituídos por duas ou mais camadas de células epiteliais de forma prismática sendo os núcleos volumosos ricos em eucromatina e nucléolos evidentes. Os alvéolos mamários eram numerosos, estando agrupados formando lóbulos mamários (Figura 1 - ECE).

\section{Morfométricos}

Os resultados morfométricos estão expressos na Tabela 2. Observar que o grupo ECE apresentou maior número de alvéolos por $\mathrm{mm}^{2}$ $(12,3 \pm 7,1 ; p<0,05 \%)$ do que os outros grupos $(1,4 \pm 2,1$ 
no CON e 1,6 $\pm 3,8$ no ISO), sendo o volume dessas células maior $\left(\mathrm{ECE}=27,4 \pm 9,7 \mu \mathrm{m}^{3}, \mathrm{p}<0,05 \%\right)$ do que nos grupos COM $\left(14,9 \pm 4,9 \mu \mathrm{m}^{3}\right)$ e tratado com ISO $\left(11,4 \pm 6,9 \mu \mathrm{m}^{3}\right)$, o mesmo ocorrendo em relação ao número de vasos sanguíneos por $\mathrm{mm}^{2}[16,4 \pm 1,5$

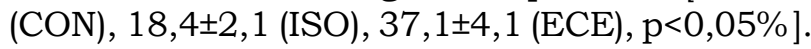

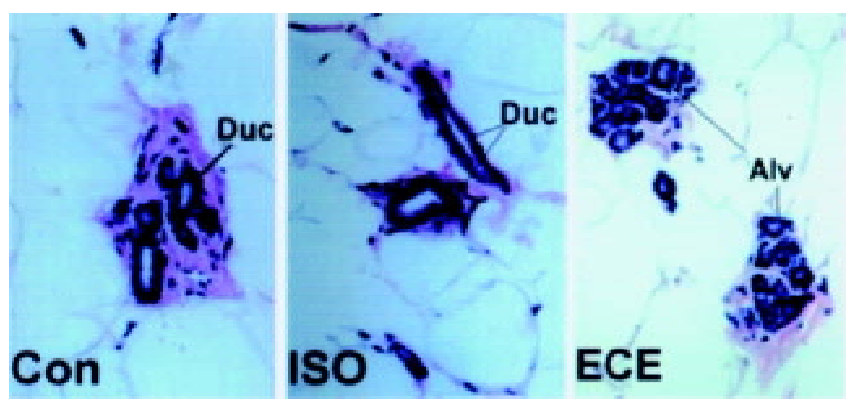

Figura 1 - Fotomicrografias de porção do tecido mamário de ratas pertencentes aos vários grupos de estudo. CON - grupo controle, ISO - grupo tratado com isoflavonas e ECE - grupo tratado com estrogênios conjugados eqüinos. Duc = ducto mamário, Alv = alvéolo. $\mathrm{HE}=150 \mathrm{X}$.

Tabela 2 - Dados morfométricos do tecido mamário de ratas castradas controle (CON) tratadas com o veículo dos fármacos (propilenoglicol), com isoflavonas (ISO) ou um conjugado de estrogênios eqüinos (ECE) durante 60 dias (média \pm desvio-padrão).

\begin{tabular}{|c|c|c|c|}
\hline Grupo & CON & ISO & ECE \\
\hline Parâmetro & (veículo) & (isoflavonas) & (estrogênios) \\
\hline Número de alvéolos $/ \mathrm{mm}^{2}$ & $1,4 \pm 2,1$ & $1,6 \pm 3,8$ & $12,3 \pm 7,1^{*}$ \\
\hline $\begin{array}{l}\text { Volume das células dos } \\
\text { alvéolos }\left(\mu \mathrm{m}^{3}\right)\end{array}$ & $14,9 \pm 4,9$ & $11,4 \pm 6,9$ & $27,4 \pm 9,7^{*}$ \\
\hline Número de vasos $/ \mathrm{mm}^{2}$ & $16,4 \pm 1,5$ & $18,4 \pm 2,1$ & $37,1 \pm 4,1^{*}$ \\
\hline
\end{tabular}

\section{RT-PCR para o VEGF}

Os resultados da eletroforese em gel de agarose dos produtos do RT-PCR para o VEGF e para a beta-actina estão expressos na Figura 2. Em relação à expressão do VEGF, o grupo ECE apresentou maior positividade de expressão do que os grupos CON e o tratado com ISO, sendo que nos grupos CON e ISO a marcação mostrou-se semelhante.

\section{Discussão}

As isoflavonas correspondem à mistura de substâncias que têm diferentes afinidades pelos receptores de estrogênio, dentre essas, destacase a genisteína, por apresentar maior atividade estrogênica ${ }^{15}$. Em trabalho anterior utilizando o mesmo tipo de isoflavonas determinou-se que a dose de $100 \mathrm{mg} / \mathrm{kg}$ foi suficiente para aumentar significantemente o peso do útero em ratas castradas ${ }^{13}$. Como controle positivo no presente trabalho utilizamos estrogênios conjugados eqüinos ${ }^{14}$.

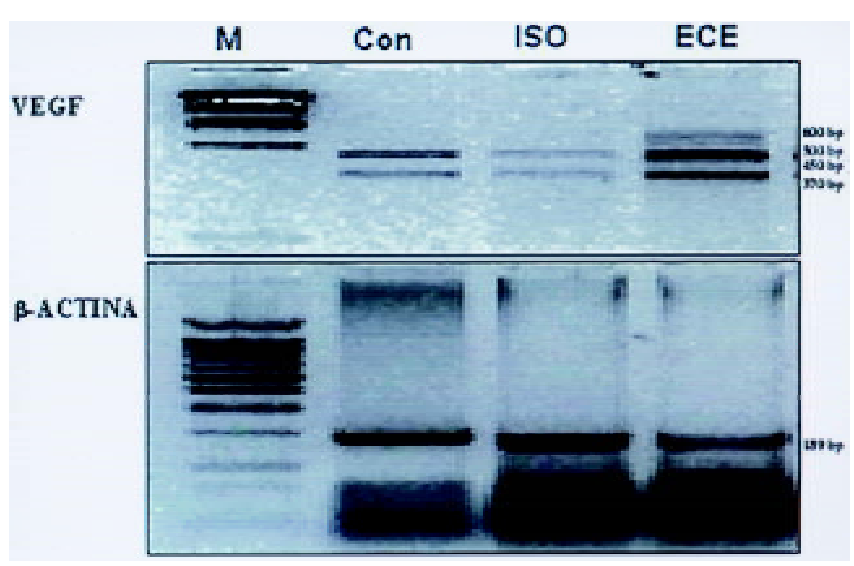

Figura 2 - Eletroforese da expressão do RNAm do gene VEGF e da $\beta$-actina na mama de ratas castradas (CON), tratadas com isoflavona (ISO) ou tratadas com estrogênios conjugados eqüinos (ECE). $M=$ marcador.

Alguns autores mostraram que a genisteína seria um preventivo para o desenvolvimento do câncer de mama por apresentar propriedades antiproliferativas ${ }^{16}$, ao passo que outros mostraram que este fito-hormônio estimularia o crescimento de tumores estrogênio-dependentes ${ }^{17}$. Possivelmente, este efeito estaria na dependência da dose utilizada, pois experimentos in vitro mostraram que a genisteína em concentrações menores que $10 \mu \mathrm{mol} / \mathrm{L}$, em células de câncer de mama humana estrogênio-dependente (MCF-7), aumentaria a expressão do RNA mensageiro do gene pS2 (marcador de proliferação). Já concentrações maiores de genisteina $(>10 \mu \mathrm{mol} / \mathrm{L})$ teriam ação contrária, inibindo a proliferação das células MCF- $7^{18}$.

Estudos realizados em ratas atímicas e ooforectomizadas que consumiram dieta enriquecida com genisteína (750 ppm) por cinco dias consecutivos mostraram aumento do número e do tamanho dos ductos e das glândulas mamárias ${ }^{11}$. Em outro experimento, utilizando ratas adultas ooforectomizadas implantadas com células de câncer de mama humano (MCF-7), também se mostrou proliferação celular com alimentos ricos em genisteína ${ }^{19}$. Além disso, outros estudos com células mamárias relataram propriedades antiproliferativas da genisteína, como modulador seletivo do receptor de estrogênio tipo beta, bem como ação antioxidante, indução da diferenciação e modulação da apoptose, inibição da topoisomerase II, do fator de crescimento tumoral beta $1 \mathrm{e}$ do fator de crescimento epidérmico ${ }^{20}$. Reforçando 
esses estudos, experimentos relatam que dieta baseada em soja inibiria o crescimento do câncer mamário induzido por carcinogênios ${ }^{21}$, ao passo que outros experimentos mostraram redução do número de tumores mamários em ratas ooforectomizadas após administração de genisteína.

Hawrylewicz et al. ${ }^{21}$ sugerem que este hormônio atuaria na diferenciação celular. Contudo, este efeito desaparece após longo tempo de administração de genisteína. No nosso estudo, as isoflavonas não mostraram efeitos significantes na proliferação mamária (número de alvéolos/ $\mathrm{mm}^{2}$, volume das células alveolares e número de vasos sanguíneos $/ \mathrm{mm}^{2}$ ) quando ratas adultas foram tratadas por periodo de 60 dias. Talvez outros estudos sejam necessários, talvez por periodo mais prolongado, para avaliar os efeitos tardios desse composto no tecido mamário.

O fator de crescimento endotelial vascular (VEGF) é uma família de glicoproteínas que induzem proliferação vascular ${ }^{22}$, com pelo menos quatro subtipos (A, B, C e D), dos quais o mais bem estudado é o VEGF-A, crítico para o desenvolvimento embrionário, e que está envolvido na maioria dos processos angiogênicos. O VEGF-B parece estar relacionado com as coronárias. O VEGF-C está implicado na formação dos vasos linfáticos e o VEGF-D é fator ainda pouco estudado ${ }^{23}$. Em nosso estudo, foi utilizado o VEGF-A, que está relacionado com o processo de angiogênese e intimamente relacionado com os processos proliferativos. Como controle positivo indicativo da presença do RNA na amostra utilizamos o RT-PCR para a beta-actina, a qual está presente no citoesqueleto de todas as células de mamíferos. É conhecido que o VEGF-A é influenciado pelos estrogênios em tecidos hormônio-dependentes ${ }^{24}$. Em nosso experimento, nota-se que os estrogênios conjugados eqüinos estimularam esse fator na mama de ratas, o mesmo não ocorrendo com as isoflavonas na dose administrada.

Há outros autores que contestam a ação protetora das isoflavonas. Estes advogam que mulheres com dieta rica em isoflavonas (altas doses) poderiam ter proliferação de lesões pré-malignas e aumento de risco de câncer de mama, sendo que o tempo de exposição seria o determinante para este efeito ${ }^{12}$. Contudo, outros estudos não comprovaram esta hipótese, nem os nossos resultados sugeriram ação proliferativa desses hormônios no tecido mamário.

No nosso estudo, deve ser ressaltado que o composto de isoflavonas tem, além da genisteína outras substâncias que, por terem semelhança estrutural e funcional com os estrogênios, poderiam modular o receptor de estrogênio ${ }^{25,26}$. No entanto, nossos resultados não evidenciaram efeito proliferativo no tecido mamário de ratas castradas quando submetidas por 60 dias consecutivos a tratamento com isoflavonas.

$\mathrm{O}$ real mecanismo pelo qual as isoflavonas influenciam este tecido ainda é controverso. Apesar de os nossos resultados não mostrarem efeito trófico na mama de ratas, recomendam-se ainda os mesmos cuidados durante o seu uso para o rastreamento do câncer de mama que se preconizam na vigência de tratamento com estrogênios.

\section{Referências}

1. Han K, Soares JM Jr, Haidar MA, de Lima GR, Baracat EC. Benefits of soy isoflavone therapeutic regimen on menopausal symptoms. Obstet Gynecol. 2002;99(3):389-94.

2. Baracat EC, Soares Júnior JM, Haidar MA, Rodrigues de Lima G. Aspectos reprodutivos no climatério. In: Fernandes CE, coordenador. Consenso Brasileiro Multidisciplinar de Assistência à Saúde da Mulher Climatérica. São Paulo: SOBRAC; Rio de Janeiro: FEBRASGO; 2003. p. 251-3.

3. Grady D, Yaffe K, Kristof M, Lin F, Richards C, Barrett-Connor E. Effect of postmenopausal hormone therapy on cognitive function: the Heart and Estrogen/progestin Replacement Study. Am J Med. 2002;113(7):543-8.

4. Zandi PP, Carlson MC, Plassman BL, Welsh-Bohmer KA, Mayer LS, Steffens DC, et al. Hormone replacement therapy and incidence of Alzheimer disease in older women: the Cache County Study. JAMA. 2002;288(17):2123-9.

5. Manson JE, Hsia J, Johnson KC, Rossouw JE, Assaf $\mathrm{AR}$, Lasser NL, et al. Estrogen plus progestin and the risk of coronary heart disease. $\mathrm{N}$ Engl $\mathrm{J}$ Med. 2003;349(6):523-34.

6. Chlebowski RT, Hendrix SL, Langer RD, Stefanick ML, Gass M, Lane D, et al. Influence of estrogen plus progestin on breast cancer and mammography in healthy postmenopausal women: the Women's Health Initiative Randomized Trial. JAMA. 2003;289(24):3243-53.

7. Knight DC, Eden JA. A review of the clinical effects of phytoestrogens. Obstet Ginecol. 1996;87(5 Pt 2):897-904.

8. The role of isoflavones in menopausal health: consensus opinion of The North American Menopause Society. Menopause. 2000;7(4):215-29.

9. Somjen D, Stern N, Knoll E, Sharon O, Gayer B, Kulik T, et al. Carboxy derivates of isoflavones as affinity carriers for cytotoxic drug targeting in adrenocortical H295R carcinoma cells. J Endocrinol. 2003;179(3):395-403. 
10. Schleicher RL, Lamartiniere CA, Zheng M, Zhang M. The inhibitory effect of genistein on the growth and metastasis of a transplantable rat accessory sex gland carcinoma. Cancer Lett. 1999;136(2):195201.

11. Wu AH, Stanczyk FZ, Hendrich S, Murphy PA, Zhang C, Wan P, et al. Effects of soy foods on ovarian function in premenopausal women. $\mathrm{Br} \mathrm{J}$ Cancer. 2000;82(11):1879-86.

12. Frey RS, Li J, Singletary KW. Effects of genistein on cell proliferation and cell cycle arrest in nonneoplastic human mammary epithelial cells: involvement of Cdc2, p2 $1^{\text {waf/cip } 1}$, p2 $7^{\text {kip } 1}$, and Cdc25C expression. Biochem Pharmacol. 2001;61(8):979-89.

13. Mosquette R. Efeito imunohistoquímico, molecular e morfológico das isoflavonas no útero de ratas [tese]. São Paulo: Universidade Federal de São Paulo; 2004.

14. Baracat EC, Simões MJ, Soares JM Jr, Haidar MA, Lima GR. Determination of physiological doses of oral conjugated equine estrogens and medroxyprogesterone acetate in adult oophorectomized rats. Climateric. 1999;2(11):198.

15. Piovesan AC, Simões RS, Mosquette R, Simões MJ, Soares Júnior JM, Baracat EC. Efeitos das isoflavonas no tecido mamário. Femina. 2004;32(9):759-63.

16. Fritz WA, Coward L, Wang J, Lamartiniere CA. Dietary genistein: perinatal mammary cancer prevention, bioavailability and toxicity testing in the rat. Carcinogenesis. 1998;19(12):2151-8.

17. Hsieh CY, Santell RC, Haslam SZ, Helferich WG. Estrogenic effects of genistein on the growth of estrogen receptor-positive human breast cancer $(\mathrm{MCF}-7)$ cells in vitro and in vivo. Cancer Res. 1998;58(17):3833-8.

18.de Lemos ML. Effects of soy phytoestrogens genistein and daidzein on breast cancer growth. Ann Pharmacother. 2001;35(9):1118-21.
19. Helferich WG. Paradoxical effects of the soy phytoestrogen genistein on growth of human breast cancer cells in vitro and in vivo. Am J Clin Nutr. 1998;68(6 Suppl):1524S-1525S.

20. Ravindranath MH, Muthugounder S, Presser N, Viswanathan S. Anticancer therapeutic potential of soy isoflavone, genistein. Adv Exp Med Biol. 2004;546:121-65.

21. Hawrylewicz EJ, Huang HH, Blair WH. Dietary soybean isolate and methionine supplementation affect mammary tumor progression in rats. J Nutr. 1991;121(10):1693-8.

22. Yi XJ, Jiang HY, Lee KK, O WS, Tang PL, Chow PH. Expression of vascular endothelial growth factor (VEGF) and its receptors during embryonic implantation in the golden hamster (Mesocricetus auratus). Cell Tissue Res. 1999;296(2):339-49.

23. Nakagawa K, Chen YX, Ishibashi H, Yonemtsu Y, Murata T, Hata Y, et al. Angiogenesis and its regulation: roles of vascular endothelial cell growth factor. Semin Thromb Hemost. 2000;26(1):61-6.

24. Vaskivuo T, Ottander U, Oduwole O, Isomaa V, Vihko P, Olofsson JL, et al. Role of apoptosis, apoptosis-related factors and 17 betahydroxysteroid dehydrogenases in human corpus luteum regression. Mol Cell Endocrinol. 2002;194(1-2):191-200.

25. Morito K, Hirose T, Kinjo J, Hirakawa T, Okawa M, Nohara $\mathrm{T}$, et al. Interaction of phytoestrogens with estrogen receptors alpha and beta. Biol Pharm Bull. 2001;24(4):351-6.

26. Pike AC, Brzozowski AM, Hubbard RE, Bonn T, Thorsell AG, Engstrom O, et al. Structure of the ligand-binding domain of oestrogen receptor beta in the presence of a partial agonist and a full antagonist. EMBO J. 1991;18(17):4608-18. 